\title{
Projeção ortodôntica de incisivos inferiores: um risco à recessão periodontal?
}

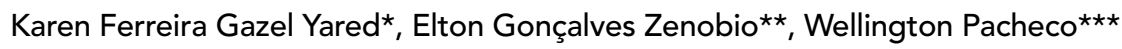

\begin{abstract}
Resumo
Para alguns casos ortodônticos, a projeção vestibular dos incisivos inferiores apresenta-se com notável significado. No entanto, após esse tipo de movimento ortodôntico, estudos observaram a ocorrência de um efeito periodontal indesejado, a recessão periodontal, em contraste a outros autores que não constataram igual associação. Diante das divergências encontradas, este estudo se propôs a analisar criticamente os resultados apresentados na literatura sobre a relação entre projeção ortodôntica e recessão periodontal vestibular em incisivos inferiores. O número de estudos sobre este tema, principalmente envolvendo adultos, é relativamente pequeno, e os resultados são conflitantes, possivelmente devido às diferenças metodológicas. Considerando-se que a recessão periodontal representa uma condição multifatorial, em que ocorre a associação entre variáveis externas e características anatômicas locais, os fatores de maior relevância detectados neste tema foram: inclinação final associada à espessura da gengiva marginal dos incisivos inferiores.
\end{abstract}

Palavras-chave: Projeção ortodôntica. Incisivos inferiores. Recessão gengival.

\section{INTRODUÇÃO}

A recessão periodontal se caracteriza por uma perda de inserção, expondo a superfície radicular, estando a margem gengival localizada apicalmente à junção cemento-esmalte ${ }^{1}$. Os problemas clínicos associados incluem: sensibilidade dentinária, estética desagradável, perda de suporte periodontal, dificuldade da manutenção da higiene bucal, dificuldade no sucesso do reparo periodontal e aumento do risco de lesão de cárie na região.

Os estudos sobre a etiologia da recessão periodontal têm demonstrado a importância da presença de fatores como: biofilme bacteriano dentário e inflamação gengival, oclusão trauma- togênica, trauma proveniente da escovação ou da inserção alterada do freio labial e características anatômicas locais relacionadas ao posicionamento dentário, largura da faixa de mucosa ceratiniza$\mathrm{da}$, espessura da gengiva marginal e tecido ósseo subjacente ${ }^{3,13,15,27}$.

A recessão periodontal envolvendo a região vestibular de incisivos inferiores tem sido alvo de constantes discussões na literatura. Tal área apresenta uma das maiores ocorrências de recessão ${ }^{1}$, estando entre as menores faixas de gengiva ceratinizada da cavidade bucal ${ }^{16}$ e apresentando cobertura óssea vestibular à raiz bastante limitada em espessura ${ }^{22}$. Dessa forma, assim como o

* Mestre em Ortodontia COP - PUCMINAS.

** Doutor em Periodontia FOA-UNESP. Professor Adjunto III Periodontia PUCMINAS. Coordenador área de Periodontia, Mestrado COP- PUCMINAS.

*** Mestre em Ortodontia UFRJ. Professor Adjunto III do Mestrado em Ortodontia COP - PUCMINAS 
posicionamento vestibular, a projeção ortodôntica dos incisivos inferiores vem sendo investigada como uma possivel precursora para o desenvolvimento da recessão periodontal ${ }^{2,5,8}$. Entretanto, não existe na literatura um consenso sobre esta questão, sendo o número reduzido e os resultados conflitantes entre os estudos.

Assim, por meio da análise crítica da literatura, este estudo teve como objetivo avaliar o estado atual no que se refere à relação entre a projeção ortodôntica de incisivos inferiores e o desenvolvimento da recessão periodontal.

\section{REVISÃO DE LITERATURA}

A literatura ortodôntica apresenta inúmeras tentativas de padronização da posição e inclinação dos incisivos inferiores, especialmente quando considerada a questão da estética facial ${ }^{23,25}$. Entretanto, a dependência da inclinação do incisivo inferior, de acordo com a relação entre as bases ósseas, torna as medidas apenas como guias, sem determinar uma regra nos objetivos de tratamento. Todavia, alguns autores consideram que determinada posição dos incisivos inferiores deve ser mantida para se obter o sucesso estético do tratamento. Desta forma, a indicação da projeção seria apenas para os casos em que esses dentes estivessem aquém da posição preconizada ${ }^{10,21}$.

Por outro lado, a projeção dos incisivos inferiores pode ser necessária, como nos casos de descompensação dentária, nos tratamentos orto-cirúrgicos de más oclusões de Classe III. Em outros, pode representar uma alternativa ao ganho de espaço para correção do apinhamento ântero-inferior, em casos de giros, desnivelamentos e alterações dentárias em sentido vestíbulo-lingual ${ }^{7,14}$. Alternativa também para a compensação da discrepância esquelética de Classe II sem possibilidade cirúrgica, representando inclusive um dos efeitos dentoalveolares de aparelhos que promovem o avanço mandibular para correção dessa má oclusão, especialmente quando a fase de crescimento já não é tão pronunciada ${ }^{20}$. Cita-se ainda a projeção que ocorre durante a fase ortodôntica de nivelamento da curva de Spee, com conseqüente correção do trespasse vertical anterior ${ }^{17}$. Assim sendo, a projeção dos incisivos inferiores representa uma realidade na clínica ortodôntica.

Viazis, Corinaldesi, Abramson ${ }^{27}$; Andlin-Sobocki e Bodin ${ }^{3}$ destacaram a ausência de consenso na literatura sobre a influência do movimento ortodôntico sagital anterior dos incisivos inferiores na recessão periodontal, tendo em vista os diferentes resultados relatados na literatura até então.

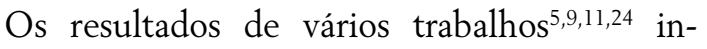
dicaram o aumento da ocorrência e gravidade da recessão com o aumento da quantidade total de projeção ortodôntica dos incisivos inferiores. Contrariamente, outros trabalhos ${ }^{2,4,8,19,22,31}$ não encontraram correlação entre tais variáveis.

Há de se frisar, contudo, as limitações e diferenças metodológicas dos estudos citados, o que dificultou qualquer conclusão geral e definitiva sobre o assunto.

\section{Presença de correlação entre projeção e re- cessão periodontal dos incisivos inferiores}

Dorfman $^{9}$ trabalhou com pacientes adolescentes, selecionados a partir da observação da presença da altura da faixa de mucosa ceratinizada inicial menor que $2 \mathrm{~mm}$, restringindo as conclusões sobre recessão e projeção a pacientes com tal característica e nessa faixa etária. Além disso, a medida da recessão foi realizada em slides e não clinicamente, o que diminuiu a precisão da avaliação.

Em relação aos estudos de Steiner, Pearson , Ainamo ${ }^{24}$; Engelking e Zachrisson ${ }^{11}$, todos os resultados foram baseados em uma amostra de macacos. Entretanto, algumas observações em animais podem ser úteis na tentativa de explicar alguns processos semelhantes nos seres humanos. Steiner, Pearson e Ainamo ${ }^{24}$ sugeriram a hipótese de um possivel afinamento da gengiva vestibular, sob a tensão relativa à projeção ortodôntica, resultar na recessão. Engelking e Zachrisson ${ }^{11}$ detectaram, na mesma amostra, que a recessão aumentou com o 
tempo, alertando para a necessidade de avaliá-la em um prazo maior e não no momento da remoção do aparelho.

Dos trabalhos encontrados sobre o tema, o estudo de Artun e Krogstad ${ }^{5}$ foi um dos que apresentou maior confiabilidade metodológica. Salienta-se, também, o fato de ter sido o único trabalho prévio que incluiu somente pacientes adultos, avaliados em um tempo maior pós-tratamento, e não apenas no momento da remoção do aparelho, e que realizaram a avaliação clínica da recessão e da saúde periodontal dos incisivos inferiores. Esse representou um estudo retrospectivo, sendo a casuística composta por pacientes orto-cirúrgicos, portadores de má oclusão de Classe III, com limite etário máximo de 41 anos, restrigindo os resultados a adultos com tais características. É importante lembrar que Handelman ${ }^{15}$ encontrou, em más oclusões de Classe III, sem excessos faciais verticais, um padrão de espessura da sínfise mais fina do que em comparação com as más oclusões de Classe I e II sem alterações verticais. Contrariando Engelking e Zachrisson ${ }^{11}$, os autores verificaram que a recessão ocorre principalmente em curto prazo, ou seja, no período ativo de tratamento e nos primeiros períodos de acompanhamento, sendo seu aumento desprezível com o tempo. Isto significa que, uma vez que a deiscência óssea tenha sido eliminada e a distância normal entre os tecidos de inserção e ósseo seja restabelecida, não há progressão da recessão. A justificativa para as contradições está no que foi considerado como "longo prazo" ou como "aumento da recessão com o tempo". O período de avaliação de Engelking e Zachrisson $^{11}$ foi de 8 meses após o tratamento, enquanto o de Artun e Krogstad ${ }^{5}$ foi de 3 anos e depois de 7 a 8 anos pós-tratamento. Cita-se novamente que a resposta do estudo de Engelking e Zachrisson ${ }^{11}$ foi proveniente de uma amostra de animais.

O estudo de Batenhorst, Bowers e Williams ${ }^{6}$ também realizado com animais, não permitiu maior elucidação sobre o tema, tendo ocorrido um grande movimento de extrusão concomitante com a projeção durante o experimento, o qual influenciou os resultados encontrados.

Em se tratando dos estudos que não encontraram correlação entre projeção ortodôntica e recessão periodontal nos incisivos inferiores, serão discutidas a seguir suas características principais.

\section{Ausência de correlação entre projeção e re- cessão periodontal dos incisivos inferiores}

Pearson ${ }^{19}$ estudou apenas pacientes com recessão, o que impossibilitou avaliar a relação direta da mesma com a projeção. Além disso, não houve restrição à faixa etária estudada, e as medidas da recessão realizadas pela altura da coroa clínica dos incisivos inferiores nos modelos de estudo, sem considerar a exposição radicular e da junção cemento-esmalte. Não havendo avaliação clínica, não pôde ser detectada a condição de saúde periodontal do paciente.

O estudo de Wingard e Bowers ${ }^{31}$, assim como de Steiner, Pearson, Ainamo ${ }^{24}$; Engelking, Zachrisson ${ }^{11}$, também foi realizado com macacos, apresentando, portanto, as mesmas limitações dos experimentos com animais. De qualquer forma, comparando-se o primeiro com os dois últimos estudos, o mesmo é criticado pelos próprios autores, devido à pequena projeção obtida dos incisivos inferiores e o curto período de avaliação que poderiam ter influenciado no resultado da ausência de correlação entre a quantidade de projeção e a ocorrência de deiscências, fenestrações ósseas e alterações gengivais.

Analisando os trabalhos de Ruf, Hansen, Pancherz $^{22}$; Djeu, Hayes e Zawaideh ${ }^{8}$, observa-se novamente a medição da recessão apenas por meio de slides e modelos de estudo, não ocorrendo a avaliação periodontal clínica, nem o conhecimento sobre a condição de saúde periodontal dos pacientes. Além disso, a única referência de recessão após a projeção ocorreu no momento final ao tratamento ortodôntico, não havendo avaliação em um período maior. Em relação à faixa etária, o primeiro autor incluiu somente pacientes 
adolescentes na casuística, enquanto no segundo estudo participaram pacientes de quaisquer idades. Outra característica relevante do estudo de Djeu, Hayes e Zawaideh ${ }^{8}$ foi que somente pequenas projeções dos incisivos inferiores foram obtidas (menores que $2^{\circ}$ ), dificultando portanto conclusões a respeito de projeções maiores.

Em relação ao estudo de Artun e Grobéty ${ }^{4}$, os autores realizaram estudo semelhante ao de Artun e $\operatorname{Krogstad}^{5}$, porém com pacientes adolescentes, sendo também retrospectivo, com avaliação clínica periodontal dos pacientes de 7,83 anos após o tratamento ortodôntico. Da mesma forma, os autores também detectaram que a recessão periodontal após o tratamento ortodôntico não é progressiva. Todavia, essa observação foi baseada na comparação da medição da recessão realizada em slides, ao final do tratamento, e na realizada no exame clínico, no período de acompanhamento.

Por último, cita-se o estudo recente de Allais e Melsen², que representou o segundo sobre o tema, envolvendo somente pacientes adultos. Ao contrário de Artun e Krogstad ${ }^{5}$, os autores não realizaram a avaliação clínica periodontal para mensuração da recessão e determinação da condição de saúde periodontal da casuística. Além disso, avaliaram a recessão após a projeção somente no momento final do tratamento e não em um período maior. A casuística não foi composta apenas por adultos jovens, sendo que o limite etário incorporado excedeu os 60 anos, apresentando, portanto, uma ampla faixa de variação nas idades. De qualquer forma, os resultados mostraram que o grupo com projeção apresentou média e número de recessões levemente maiores que o grupo controle, mas esses dados foram considerados irrelevantes clínica e estatisticamente. Um dado importante do estudo foi a avaliação da movimentação dos incisivos inferiores exclusivamente por modelos de estudo, o que o diferenciou dos demais. A medição foi realizada a partir dos pontos de contato mesiais dos primeiros molares inferiores ao ponto de contato entre os incisivos centrais inferiores, representando o comprimento do arco. Entretanto, tal medição não indica precisamente o movimento dos quatro incisivos inferiores, conforme foi considerado. Por exemplo, incisivos centrais inferiores girados, com as superfícies mesiais lingualizadas, mas com espaço presente no arco, podem ter sido apenas rotacionados em torno de seus centros de resistência durante o tratamento, sem haver projeção verdadeira desses dentes, e principalmente dos incisivos laterais. Portanto, a medida do comprimento do arco realizada dessa forma indicaria uma falsa projeção. O ideal nesse caso é que as medidas tivessem sido obtidas para cada um dos quatro incisivos inferiores, no centro da borda incisal.

\section{Considerações ortodônticas / periodontais}

Ressalta-se a importância do conhecimento da condição de saúde periodontal da casuística, o que não foi avaliado na maioria dos estudos revistos, a fim de permitir conclusões fundamentadas sobre o tema, lembrando-se que a recessão periodontal pode ter origem multifatorial. Da mesma forma, nenhum dos estudos considerou fatores como oclusão traumatogênica, inserção alta do freio labial inferior, dimensões mucogengivais e inclinação final dos incisivos inferiores.

Geiger ${ }^{13}$ confirmou essas considerações, justificando por que alguns casos isolados desenvolvem recessão, enquanto outros, que se submeteram à expansão anterior acentuada dos incisivos inferiores e sem controle de placa, nada apresentam. Na verdade, no primeiro caso a projeção ocorreria contra áreas com deficiência de gengiva inserida, oferecendo menor resistência à tensão. Esse conceito é suportado pelos casos de recessão em que a gengiva remanescente se apresenta relativamente livre de placa ou inflamação. Por esse motivo, Newman, Goldman e Newman ${ }^{18}$ alertaram que áreas com altura diminuída da faixa de gengiva inserida ou exibindo alguma recessão em incisivos inferiores já projetados devem ser examinadas e monitoradas cuidadosamente antes, durante e 
após o tratamento ortodôntico, devido ao risco da recessão. Nesses casos, antes do tratamento, se prevista uma projeção do incisivo, o ortodontista deve estar a par das possíveis técnicas para a correção de um potencial problema periodontal. Por razões médico-legais, o periodontista deve ser consultado e uma declaração mantida anexa aos registros do paciente. Entretanto, segundo os autores, é importante que o planejamento do tratamento ortodôntico seja alterado se o movimento vestibular do dente puder causar um problema mucogengival que não seja passível de ser tratado periodontalmente e que gere estética insatisfatória.

Assim sendo, diante dos dados mais recentes, Vanzin et al. ${ }^{26}$, por meio de uma revisão da literatura, sugeriram que o efeito da recessão à projeção estivesse relacionado a pacientes adultos, e não a adolescentes. Provavelmente, os fatores determinantes da recessão não inflamatória envolvem características do tecido periodontal local, como a natureza mucogengival e do tecido ósseo subjacente.

A espessura óssea representa um fator importante na ocorrência da recessão periodontal quando incisivos inferiores são projetados ortodonticamente, de acordo com Handelman ${ }^{15}$. Todos os três casos de biprotrusão, ilustrados em seu estudo, apresentavam largura alveolar fina e altura facial ânteroinferior aumentada. O tratamento foi limitado pela sinfise estreita, não havendo melhora significativa do perfil. $\mathrm{O}$ autor citou ainda um caso de perfuração da cortical lingual e recessão periodontal, quando da projeção do incisivo inferior para compensar o trespasse horizontal, em uma paciente portadora de má oclusão de Classe II. Portanto, pacientes com alvéolo estreito ou discrepância esquelética severa demonstram limitação para a correção ortodôntica e podem requerer cirurgia. $\mathrm{O}$ autor afirmou que movimentar um dente ilimitadamente significa acreditar que ocorrerá reconstituição óssea inteiramente em qualquer direção para a qual o dente seja movimentado, o que deve ser reconsiderado.

Wehrbein, Bauer e Diedrich ${ }^{28}$ também avaliaram os efeitos da movimentação dentária no osso de suporte na região dos incisivos inferiores, a partir da mandíbula dissecada de uma paciente tratada ortodonticamente, que havia falecido por acidente. Os resultados mostraram que o movimento sagital dos incisivos pode levar à perda óssea na direção do movimento. Não parece que essa perda óssea seja um efeito de influências inflamatórias, segundo os autores, já que as placas corticais interproximais aos incisivos estavam normais, dentro dos limites fisiológicos. Assim sendo, a quantidade maior de osso cortical vestibular é necessária para manter a integridade da placa óssea sagital nesse tipo de movimento. Dessa forma, Wennström et al. ${ }^{29}$ sugeriram que, se o dente movimentado ortodonticamente for deslocado para fora do envelope ósseo do processo alveolar e uma deiscência permanente for estabelecida, o risco do desenvolvimento da recessão periodontal será iminente. Entretanto, Fuhrmann ${ }^{12}$ demonstrou, em um estudo com tomografia computadorizada em pacientes adultos, que deiscências e fenestrações ósseas poderiam existir antes mesmo do movimento ortodôntico vestibular dos incisivos, mesmo sem nenhum indício de recessão periodontal clinicamente perceptível. Além disso, o autor mostrou que a projeção dos incisivos poderia aumentar essas alterações ósseas.

Além da altura da faixa de mucosa ceratinizada e da espessura óssea na região dos incisivos inferiores, a espessura da gengiva marginal também foi relatada como fator de maior importância no desenvolvimento da recessão.

Wennström ${ }^{30}$ propôs que incisivos inferiores movimentados nos limites do processo alveolar não deverão apresentar recessão. Entretanto, quando movimentados com uma expansão descontrolada, além da cortical óssea, deiscências e fenestrações ósseas vestibulares poderão ser induzidas. Além disso, a projeção causaria a tensão do tecido gengival marginal livre, tornando-o mais fino tanto no sentido ápico-coronal quanto vestíbulo-lingual. Todas essas alterações, em decorrência da projeção ortodôntica, tornariam o tecido gengival vestibular vulnerável, com menor resistência à presença da 
placa e ao trauma proveniente da técnica de escovação incorreta. Dessa forma se estabeleceria um ambiente propício ao desenvolvimento da recessão periodontal. Portanto, quando se referenciar a possibilidade da ocorrência da recessão periodontal durante ou após a fase ativa da movimentação ortodôntica, os fatores importantes a se considerar são: a direção e sentido do movimento, bem como a espessura vestíbulo-lingual da gengiva. $\mathrm{O}$ autor alerta que muitos estudos sobre o assunto não consideraram a espessura da gengiva marginal, daí a variabilidade das respostas encontradas. No entanto, nenhum valor limite entre espessura favorável ou desfavorável foi comprovado.

Valores inéditos para espessura da gengiva marginal nesse tipo de estudo foram demonstrados por Yared, Zenobio e Pacheco ${ }^{32}$, que apresentaram os grupos de risco à recessão quando da projeção vestibular de incisivos centrais inferiores. $\mathrm{O}$ estudo foi composto por 34 adultos, entre 18 e 33 anos, saudáveis periodontalmente, com avaliação clínica após 7 a 47 meses do tratamento ortodôntico, e estabelecidos critérios rigorosos na obtenção da casuística. A movimentação foi avaliada por telerradiografias em norma lateral, não havendo magnificação radiográfica significativa entre as mesmas. A espessura da gengiva marginal foi medida por meio de um paquímetro digital modificado para fins próprios. Os resultados mostraram que a inclinação final, independente da quantidade total de projeção, e a espessura da gengiva marginal representaria reais fatores de relevância na relação com a recessão periodontal. A inclinação final dos incisivos centrais inferiores acima de $95^{\circ}$, associada à espessura da gengiva marginal menor que $0,5 \mathrm{~mm}$, estiveram diretamente relacionadas às maiores incidência e gravidade da recessão nesses dentes, sendo a espessura mais determinante que a inclinação.

\section{CONSIDERAÇÕES FINAIS}

- Existem controvérsias na literatura sobre a relação entre projeção ortodôntica de incisivos inferiores e o desenvolvimento da recessão periodontal. No entanto, os estudos apresentaram diferenças metodológicas significativas, como: heterogeneidade nas faixas etárias das casuísticas, a variedade da quantidade de projeção obtida e dos tratamentos ortodônticos incluídos; ausência de referências em relação à inclinação final dos incisivos inferiores; diferenças no período de avaliação pós-tratamento ortodôntico e na forma de avaliar as variáveis periodontais;

- Há uma tendência entre os autores para reconhecer as características do tecido periodontal local, como a natureza mucogengival e do tecido ósseo subjacente como os fatores determinantes da recessão não inflamatória, após projeção ortodôntica de incisivos inferiores.

Orthodontic proclination of mandibular incisors: risk to gingival recession?

\begin{abstract}
The orthodontic proclination of lower incisors is very important in some cases. However, some investigations have shown that gingival recession could occur after this type of orthodontic movement, but other authors couldn't prove this. The aim of this study was to analyze the literature finds about the relationship of lower incisors proclination and gingival recession. There are few studies about it, especially with adults and the results' disagreement is possibly due to the different methods applied. Considering that association of many external and local anatomic factors represent the etiology of gingival recession, the most important factors detected in this literature review were: final inclination and the thickness of the free gingival margin of the lower incisors.
\end{abstract}

Key words: Orthodontic proclination. Mandibular incisors. Gingival recession. 


\section{REFERÊNCIAS}

1. ALBANDAR, J. M; KINGMAN, A. Gingival recession, gingival bleeding and dental calculus in adults 30 years of age and older in the United States, 1988-1994. J Periodontol, Chicago, v. 70, no.1, p. 30-43, jan. 1999.

2. ALLAIS, D.; MELSEN, B. Does labial movement of lower incisors influence the level of the gingival margin? A case-control study of adult orthodontic patients. Eur J Orthod, London, v. 25, p. 343-352, Aug. 2003.

3. ANDLIN-SOBOCKI, A.; BODIN, L. Dimensional alterations of the gingiva related to changes of facial/lingual tooth position in permanent anterior teeth of children. J Clin Periodontol, Copenhagen, v. 20, no. 3, p. 219-224, mar. 1993.

4. ARTUN, J.; GROBÉTY, D. Periodontal status of mandibular incisors after pronounced orthodontic advancement during adolescence: a follow-up evaluation. Am J Orthod Dentofacial Orthop, St. Louis, v. 119, no. 1, p. 2-10, Jan. 2001.

5. ARTUN, J.; KROGSTAD, O. Periodontal status of mandibular incisors following excessive proclination. Am J Orthod Dentofacial Orthop, St. Louis, v. 91, no. 3, p. 225-232, Mar. 1987.

6. BATENHORST, K. F.; BOWERS, G. M.; WILLIAMS, J. E. Tissue changes resulting from facial tipping and extrusion of incisors in monkeys. J Periodontol, Chicago, v. 45, no. 9, p. 660-668, Sept. 1974.

7. BRAUN, S.; HNAT, W. Dynamic relationship of the mandibular anterior segment. Am J Orthod Dentofacial Orthop, St. Louis, v. 111, p. 518-524, 1997.

8. DJEU, G.; HAYES, C.; ZAWAIDEH, S. Correlation between mandibular central incisor proclination and gingival recession during fixed appliance therapy. Angle Orthod, Appleton, v. 72, no. 3, p. 238-245, June 2002.

9. DORFMAN, H. Mucogingival changes resulting from mandibular incisor tooth movement. Am J Orthod, St. Louis, v. 74, no. 3, p. 286-297, Sept. 1978.

10. DOWNS, W. B. Analysis of the dentofacial profile. Angle Orthod, Appleton, v. 26, p.191-212, 1956.

11. ENGELKING, G.; ZACHRISSON, B. U. Effects of incisor repositioning on monkey periodontium after expansion through the cortical plate. Am J Orthod, St. Louis, v. 82, no. 1, p. 23-32, July 1982.

12. FUHRMANN, R. Three dimensional interpretation of labiolingual bone widht of the lower incisors. Part II. J Orofacial Orthop, Munich, v. 57, no. 3, p. 168-185, June 1996.

13. GEIGER, A. M. Mucogingival problems and the movement of mandibular incisors: a clinical review. Am J Orthod, St. Louis, v. 78 , no. 5 , p. 527 , nov. 1980

14. GERMANE, N. et al. Increase in arch perimeter due to orthodontic expansion. Am J Orthod Dentofacial Orthop, St. Louis, v. 100, p. 421-427, 1991.

15. HANDELMAN, C. S. The anterior alveolus: its importance in limiting orthodontic treatment and its influence on the occurrence of iatrogenic sequelae. Angle Orthod, Appleton, v. 66, no. 2, p. 95-110, 1996.

16. LANG, N. P.; LÖE, H. The relationship between the width of keratinized gingiva and gingival health. J Periodontol, Chicago, v. 43, no. 10, p. 623-627, Oct. 1972.

17. MUTINELLI, S.; MANFREDI, M.; COZZANI, M. A mathematicgeometric model to calculate variation in mandibular arch form. Eur J Orthod, London, v. 22, p. 113-125, 2000.

18. NEWMAN, G. V.; GOLDMAN, M. J.; NEWMAN, R. A. Mucogingival orthodontic and periodontal problems. Am J Orthod Dentofacial Orthop, St. Louis, v. 105, no. 4, p. 321-327, Apr. 1994.

19. PEARSON, L. E. Gingival height of lower central incisors orthodontically treated and untreated. Angle Orthod, Appleton, v. 38, no. 4, p. 337-339, Oct. 1968.

20. PROFFIT, W. R.; PHILLIPS, C.; DOUVARTZIDIS, N. A comparision of outcomes of orthodontic and surgical-orthodontic treatment of class II malocclusion in adults. Am J Orthod Dentofacial Orthop, St. Louis, v. 101, no. 6, p. 556-565, 1992.
21. RICKETTS, R. M. Cephalometric synthesis: an exercise in starting objective and plannig treatment with tracings of thehead roentgenogram. Am J Orthod, St. Louis, v. 46, p. 647-675, 1960.

22. RUF, S.; HANSEN, K.; PANCHERZ, H. Does orthodontic proclination of lower incisors in children end adolescents cause gingival recession? Am J Orthod Dentofacial Orthop, St. Louis, v. 114, no. 1, p. 100-106, July 1998.

23. STEINER, C. C. Cephalometrics for you and me. Am J Orthod, St. Louis, v. 39, p. 729-755, 1953.

24. STEINER, G. G.; PEARSON, J. K.; AINAMO, J. Changes of the marginal periodontium as a result of labial tooth movement in monkeys. J Periondontol, Chicago, v. 52, no. 6, p. 314-320, June 1981.

25. TWEED, C. H. The Frankfort-Mandibular incisor angle (FMIA) in orthodontic diagnosis, treatment plannig and prognosis. Angle Orthod, Appleton, v. 24, p.121-169, 1954

26. VANZIN, G. D. et al. Considerações sobre recessão gengival e proclinação excessiva dos incisivos inferiores. J Brás Ortodon Ortop Facial, Curitiba, v. 8, n. 46, p. 318-325, jul./ago. 2003.

27. VIAZIS, A. D.; CORINALDESI, G.; ABRAMSON, M. M. Gingival recession and fenestration in orthodontic treatment. J Clin Orthod, Boulder, v. 24, no. 10, p. 633-636, Oct. 1990.

28. WEHRBEIN, H.; BAUER, W.; DIEDRICH, P. Mandibular incisors, alveolar bone and symphysis after orthodontic treatment: a retrospective study. Am J Orthod Dentofacial Orthop, St. Louis, v. 110, no. 3, 239-246, Sept. 1996.

29. WENNSTRÖM, J. L. et al. Some periodontal tissue reactions to orthodontic tooth movement in monkeys. J Clin Periodontol, Copenhagen, v. 14, p.121-129, Mar. 1987.

30. WENNSTRÖM, J. L. Mucogingival considerations in orthodontic treatment. Semin Orthod, Birmingham, v. 2, no. 1, p. 46-54, Mar. 1996.

31. WINGARD, C. E.; BOWERS, G. M. The effects on facial bone from facial tipping of incisors in monkeys. J Periodontol, Chicago, v. 47, no. 8, p. 450-454, Aug. 1976.

32. YARED, K. F. G.; ZENOBIO, E.; PACHECO, W. Condição periodontal de incisivos centrais inferiores, em adultos, relacionada à projeção vestibular após tratamento ortodôntico. 2004. Dissertação (Mestrado)-Centro de Odontologia e Pesquisa, Pontifícia Universidade Católica de Minas Gerais, Belo Horizonte, 2004.

\author{
Endereço de correspondência \\ Karen Ferreira Gazel Yared \\ Rua Pernambuco, 189, sala 502, bairro Funcionários \\ CEP: $30.130-150$ - Belo Horizonte/MG \\ E-mail: kgyared@yahoo.com.br
}

